\title{
Globalization Effects on Contagion Risks in Financial Markets
}

\author{
Mariya Paskaleva ${ }^{1, *}$, and Ani Stoykova ${ }^{1}$ \\ ${ }^{1}$ South-West University "Neofit Rilski”, Faculty of Economics, Department of "Finance and \\ accounting”, 60 Ivan Mihailov str., 2700 Blagoevgrad, Bulgaria
}

\begin{abstract}
.
Research background: Financial globalization has opened international capital markets to investors and companies worldwide. However, the global financial crisis has created big volatility in the stock prices that induces a restriction in the reflection of full information. We explore ten EU Member States (France, Germany, The United Kingdom, Belgium, Bulgaria, Romania, Greece, Portugal, Ireland, Spain), and the USA. The explored period is $03.03 .2003-30.06 .2016$, as it includes the effects of the global financial crisis of 2008.

Purpose of the article: To determine if there is a contagion effect between the Bulgarian stock market and the other examined stock markets during the crisis period and whether these markets are efficient.

Methods: Argument Dickey-Fuller Test, DCC-GARCH Model, Autoregressive (AR) Models, TGARCH Model, Descriptive Statistics.

Findings \& Value added: Our results show that a contagion across the Bulgarian capital market and eight capital markets exist during the global financial crisis of 2008. We register the strongest contagion effects from US and German capital markets to the Bulgarian capital market. The Bulgarian capital market is relatively integrated with the stock markets of Germany and the United States. That is the explanation of why the Bulgarian capital market is exposed to financial contagion effects from the US capital market and the capital markets of EU member states during the crisis period. We register statistically significant AR (1) for the UK, Greece, Ireland, Portugal, Romania, and Bulgaria, and we can define these global capital markets as inefficient.
\end{abstract}

Keywords: Efficient Market Hypothesis; capital markets; dynamic conditional correlations; financial contagion; globalization

JEL Classification: $C 22 ; G 01 ; G 14 ; G 15, F 65$

\footnotetext{
*Corresponding author: m.gergova@abv.bg
} 


\section{Introduction}

Financial crises are a severe phenomenon in both developed and emerging countries. However, the financial crisis has created big volatility in the stock prices that induces a restriction in the reflection of full information. Therefore, this situation is a challenge for the Efficient Market Hypothesis. According to the Efficient Market Hypothesis (EMH), stock prices should always show a full reflection of all available and relevant information and follow a random walk process. Undoubtedly, the global financial crisis of 2008 has affected the efficiency of the global capital markets and also financial activities and macroeconomic conditions. "Contagion" became the catchword for such phenomena and is now widely being used to describe the spread of financial disturbances from one country to another [1]. After the crisis of 2008, the European and US stock markets underwent large depreciation and high stock market volatility. To examine the extent of independence and a contagion relationship between these capital markets before, during, and after the crisis, we apply the DCC-GARCH model. In this article, we aim to determine whether there are contagion effects across the Bulgarian capital market and France, Germany, The United Kingdom, Belgium, Bulgaria, Romania, Greece, Portugal, Ireland, Spain and USA during the crisis period and if these markets are efficient.

\subsection{Literature Review}

Paper [2] claims that overcoming ever-increasing macroeconomic, institutional, technological, climatic, etc. challenges, complexities, and risks facing the economies and stock markets of the Western Balkan countries requires an integrated approach for active cooperation and involvement of national, regional and international stakeholders (market and institutional ones) to successfully move from status of peripheral/frontier to emerging stock markets in the Western Balkans in the medium to long term. Paper [3] intend to identify the main studies in the literature that has as main objective the analysis of the integration of financial systems. Paper [4] makes a comprehensive stock profile for four of the most popular East Asian stock exchanges-Tokyo, Hong Kong, Taiwan, and Shanghai, for the period 2007 - 2019. It is concluded that the global financial crisis of 2008 has a significant and lasting negative impact only on the price component of the stock exchange profiles, while the stock exchange activity of the studied exchanges remains completely unaffected. Paper [5] analyses the existence of long memory in return series for nine indices from Central-Eastern European and Balkan emerging markets and they prove that all indices, except Czech index, have predictable behaviour. In paper [6] the dynamic correlation between the aggregate stock returns in Indian and the US markets is explored (taken as a proxy of the global market) using AGDCC model. The authors of paper [7] examine the comovement and spillover dynamics between the Slovenian and some European stock market returns. A dynamic conditional correlation GARCH analysis is applied to returns series of representative national stock indices for the period from April 1997 to May 2010. Paper [8] claims that the global financial crisis has clearly identified and reinforced the role of financial risks. Paper [9] states that in the financial market, risk management is associated with the process of identifying individual risks, their analyzes and making investment decisions by reducing the degree of uncertainty. The authors of paper [10] use a panel VAR methodology and they find rather comovement effects in the Visegrad group member countries (the Czech Republic, Hungary, Poland and Slovakia) as they have been only marginally affected by the turmoil in the peripheral countries during the sovereign debt crisis.

In study [11] the possible contagion from West European stock markets to stock markets in Central and Eastern Europe is investigated. The author concludes that the dynamic conditional correlation (DCCs) increased steadily between 2002 and 2012, which could be 
attributed to closer financial integration. During the crisis the dynamic correlations rose substantially, which suggests some contagion. The authors of paper [12] investigate the existence of financial contagion in the European Union during the recent Global Financial Crisis (GFC) of 2007-2009 and the European Sovereign Debt Crisis (ESDC) that started in 2009. They find evidence of a non-synchronized transition of all countries to the crisis regime, in both crises. Paper [13] examine the effects of the US financial and the Eurozone debt crises on a large set of frontier stock markets. The authors of paper [14] use their approach with daily data from 1998 to 2014 and they find evidence of financial contagion for all of our examined emerging markets. Paper [15] analyzes sovereign risk shift-contagion, i.e. positive and significant changes in the propagation mechanisms, using bond yield spreads for the major eurozone countries and they find that the propagation of shocks in euro's bond yield spreads shows almost no presence of shift-contagion in the sample periods considered. Paper [16] investigates whether contagion occurred during the recent global financial crisis across European and US financial markets. The findings indicate significant evidence of contagion, especially through the channels of higher order moments. The authors of paper [17] investigate the contagion effect from the US stock market to 10 international stock markets (developing and developed markets). The results demonstrate the correlation between the US stock market and all stock markets is higher during the crisis period than during the normal period. Capital market participants are represented through their financial statements. The information in financial statements is one of the first indicators of the development of the globalized economy. The author of paper [20] presents the theoretical and applied bases of financial statements of general-purpose enterprises. The information contained in them is intended for a wide range of users, including capital market participants, and affects the efficiency of financial markets.

\section{Methodology}

In this study, we explore the following capital market indexes for the following countries: France (CAC 40), Germany (DAX), The United Kingdom (FTSE 100), Belgium (BEL- 20), Bulgaria (SOFIX), Romania (BET), Greece (ATHEX20), Portugal (PSI-20), Ireland (ISEQ20), Spain (IBEX35) and USA (DJIA). The explored period is 03.03.2003 - 30.06.2016 as it includes the effects of the financial crisis of 2008. We divide the explored period of subperiods: pre-crisis period 03.03.2003-29.12.2006; crisis period (02.01.2007-28.12.2012) and post-crisis period 03.01.2013-30.06.2016). We apply the Argument Dickey-Fuller test to estimate stationarity. We prove that all variables are stationary in the form dlog (x) i.e. variables were integrated of order 1 .

\subsection{DCC-GARCH Model}

In the current research we apply DCC-GARCH model, which estimates conditional correlations in Multivariate GARCH models. The multivariate model is defined by the following formula:

Where:

$$
\mathbf{X}_{\mathrm{t}}=\mu_{\mathrm{t}}+\epsilon_{\mathrm{t}}
$$

$\mathbf{X}_{\mathbf{t}}=\left(\mathbf{X}_{\mathbf{1 t}}, \mathbf{X}_{\mathbf{2 t}}, \ldots \mathbf{X}_{\mathbf{N t}}\right)-$ is the vector of past observations;

$\mu_{t}=\mu_{1 t}+\mu_{2 t}+\cdots+\mu_{N t^{-}}$is the vector of conditional returns;

$\boldsymbol{\epsilon}_{\mathrm{t}}=\boldsymbol{\epsilon}_{1 \mathrm{t}}+\boldsymbol{\epsilon}_{2 \mathrm{t}}+\cdots+\boldsymbol{\epsilon}_{\mathrm{Nt}}-$ is the vector of standardized residuals.

To examine the contagion effect, we apply developed methodology by paper [18] and paper [19]. We denote the stock return of Bulgarian index with $X_{t}$ and with $\mathrm{y}_{t}-$ the stock returns of the other examined indices, respectively. 
where $\alpha$ и $\beta$ are constants and $\epsilon_{t}$ - error terms.

The correlation coefficient $\rho$ between $X_{t}$ and $Y_{t}$ is calculated by the following:

$$
\rho^{*}=\frac{\rho}{\sqrt{1+\delta\left(1-\rho^{2}\right)}}
$$

with $\delta=\frac{\sigma_{x}^{c}}{\sigma_{x}^{t}-1}$

where $\delta$ - measures the relative increase in the volatility of $X_{t}$ cross the crisis and pre-crisis period; $\sigma_{x}^{c}$-conditional variances of $X_{t}$ during the crisis period; $\sigma_{x}^{t}$ - conditional variances of $X_{t}$ during the pre-crisis period.

Following the methodology of paper [19], we develop two hypotheses. Null hypothesis $\left(\mathrm{H}_{0}\right)$ claims that the correlation between two markets does not significantly change during the crisis compared to the not-crisis period, and we can conclude that there is no financial contagion. The alternative hypothesis states that the correlation between two capital markets changes significantly across the not-crisis and crisis periods as a result of the crisis developed in one of them. Therefore, we can suggest that part of the change in volatility of the Bulgarian stock market is a result of the volatility of another market. Consequently, we can conclude that there is a financial contagion:

$H_{0}: \rho_{c}^{*}=\rho_{t}^{*}$

$H_{1}: \rho_{c}^{*}>\rho_{t}^{*}$

To reject or accept one of these hypotheses, we apply the t-Student test.

$t=\left(\rho_{c}^{*}-\rho_{t}\right) \sqrt{\frac{n_{c}+n_{t}-4}{1-\left(\rho_{c}^{*}-\rho_{t}\right)^{2}}}$

\subsection{Autoregressive (AR) Models}

An autoregressive model of order $p$, denoted AR $(p)$ has the form:

$$
Y_{t}=\rho_{1} Y_{t-1}+\rho_{2} Y_{t-2}+\ldots \rho_{p} Y_{t-p}+\varepsilon_{t}=\sum_{i=1}^{p} \rho_{j} Y_{t-j}+\varepsilon_{t}
$$

\subsection{The Threshold GARCH (TGARCH) Model}

The generalized specification for the conditional variance in TARCH or Threshold ARCH and Threshold GARCH is given by:

$$
\sigma_{t}^{2}=\omega+\sum_{j=1}^{q} \beta_{j} \sigma_{t-j}^{2}+\sum_{i=1}^{p} \quad \alpha_{i} \varepsilon_{t-i}^{2}+\sum_{k=1}^{r} \gamma_{k} \varepsilon_{t-k}^{2} I_{t-k}
$$

\section{Methodology}

During the full-time period, for mean, most of the data has a positive return except for BEL20 (Table 1). For Skewness, we found that all indices do not equal to zero, so this indicates asymmetry for all the series. The Kurtosis is greater than 3 for all of the explored countries, indicating that their distributions are leptokurtic. The normality hypothesis is rejected by the Jarque-Bera test. From the values of St. Dev., we prove that during the full explored period, the Romanian stock market is the riskiest one. We should mention that the values of the St. Dev., of the Greek and The Bulgarian capital markets are almost equal to the one of the Romanian stock markets. The standard deviation of the returns of the indices during the crisis period is higher than the stable periods. This reflects an increase in volatility during the crisis for all of the explored European capital markets. 
Table 1. Descriptive statistic of the explored indices

\begin{tabular}{|c|c|c|c|c|c|c|c|c|c|c|c|}
\hline \multicolumn{12}{|c|}{ The Full Explored Period: March 2003 - June 2016} \\
\hline & $\begin{array}{c}\text { RATH } \\
\text { EX } \\
\end{array}$ & $\begin{array}{l}\text { RBE } \\
\text { L20 } \\
\end{array}$ & $\begin{array}{c}\text { RBE } \\
\text { T }\end{array}$ & $\begin{array}{c}\text { RCA } \\
\text { C40 } \\
\end{array}$ & $\begin{array}{c}\text { RDA } \\
\mathbf{X}\end{array}$ & $\begin{array}{c}\text { RDJI } \\
\text { A }\end{array}$ & $\begin{array}{l}\text { RFTS } \\
\text { E100 }\end{array}$ & $\begin{array}{c}\text { RIBE } \\
\text { X35 }\end{array}$ & $\begin{array}{c}\text { RISE } \\
\mathbf{Q}\end{array}$ & $\begin{array}{c}\text { RPSI } \\
20 \\
\end{array}$ & $\begin{array}{c}\text { RSOF } \\
\text { IX }\end{array}$ \\
\hline Mean & $\begin{array}{l}0.002 \\
962\end{array}$ & $\begin{array}{c}- \\
0.000 \\
446 \\
\end{array}$ & $\begin{array}{l}0.008 \\
515\end{array}$ & $\begin{array}{c}0.003 \\
028\end{array}$ & $\begin{array}{l}0.008 \\
736\end{array}$ & $\begin{array}{c}0.005 \\
082\end{array}$ & $\begin{array}{c}0.0036 \\
97\end{array}$ & $\begin{array}{l}0.002 \\
074\end{array}$ & $\begin{array}{l}0.002 \\
277\end{array}$ & $\begin{array}{l}0.001 \\
653\end{array}$ & $\begin{array}{c}0.004 \\
322\end{array}$ \\
\hline $\begin{array}{l}\text { Media } \\
\text { n }\end{array}$ & $\begin{array}{c}- \\
0.0029 \\
69\end{array}$ & $\begin{array}{l}0.000 \\
176\end{array}$ & $\begin{array}{l}0.015 \\
797\end{array}$ & $\begin{array}{l}0.010 \\
546\end{array}$ & $\begin{array}{l}0.019 \\
494\end{array}$ & $\begin{array}{l}0.007 \\
609\end{array}$ & $\begin{array}{c}0.0079 \\
70\end{array}$ & $\begin{array}{l}0.008 \\
008\end{array}$ & $\begin{array}{l}0.008 \\
432\end{array}$ & $\begin{array}{c}- \\
0.001 \\
335\end{array}$ & $\begin{array}{l}0.002 \\
388\end{array}$ \\
\hline $\begin{array}{l}\text { Maxim } \\
\text { um }\end{array}$ & $\begin{array}{c}0.663 \\
535\end{array}$ & $\begin{array}{l}0.073 \\
016\end{array}$ & $\begin{array}{c}0.257 \\
241 \\
\end{array}$ & $\begin{array}{c}0.120 \\
462 \\
\end{array}$ & $\begin{array}{c}0.191 \\
631 \\
\end{array}$ & $\begin{array}{c}0.091 \\
161 \\
\end{array}$ & $\begin{array}{c}0.0830 \\
00\end{array}$ & $\begin{array}{c}0.153 \\
789\end{array}$ & $\begin{array}{l}0.178 \\
253 \\
\end{array}$ & $\begin{array}{l}0.370 \\
302\end{array}$ & $\begin{array}{c}0.251 \\
207 \\
\end{array}$ \\
\hline $\begin{array}{l}\text { Minim } \\
\text { um }\end{array}$ & $\begin{array}{c}- \\
0.3554 \\
81 \\
\end{array}$ & $\begin{array}{c}- \\
0.048 \\
918\end{array}$ & $\begin{array}{c}- \\
0.414 \\
192 \\
\end{array}$ & $\begin{array}{c}- \\
0.145 \\
225 \\
\end{array}$ & $\begin{array}{c}- \\
0.224 \\
954\end{array}$ & $\begin{array}{c}- \\
0.151 \\
526 \\
\end{array}$ & $\begin{array}{c}- \\
0.1395 \\
36\end{array}$ & $\begin{array}{c}- \\
0.1867 \\
27\end{array}$ & $\begin{array}{c}- \\
0.235 \\
823 \\
\end{array}$ & $\begin{array}{c}- \\
0.202 \\
173 \\
\end{array}$ & $\begin{array}{c}- \\
0.476 \\
323 \\
\end{array}$ \\
\hline $\begin{array}{l}\text { Std. } \\
\text { Dev. }\end{array}$ & $\begin{array}{c}0.087 \\
514 \\
\end{array}$ & $\begin{array}{l}0.015 \\
765\end{array}$ & $\begin{array}{l}0.088 \\
261 \\
\end{array}$ & $\begin{array}{c}0.047 \\
970 \\
\end{array}$ & $\begin{array}{l}0.055 \\
599\end{array}$ & $\begin{array}{l}0.038 \\
350\end{array}$ & $\begin{array}{c}0.0378 \\
10 \\
\end{array}$ & $\begin{array}{l}0.056 \\
205 \\
\end{array}$ & $\begin{array}{c}0.058 \\
590\end{array}$ & $\begin{array}{l}0.050 \\
531\end{array}$ & $\begin{array}{c}0.086 \\
087\end{array}$ \\
\hline $\begin{array}{l}\text { Skewn } \\
\text { ess }\end{array}$ & $\begin{array}{l}2.134 \\
574\end{array}$ & $\begin{array}{l}0.285 \\
622\end{array}$ & $\begin{array}{c}- \\
0.979 \\
921\end{array}$ & $\begin{array}{c}- \\
0.562 \\
221\end{array}$ & $\begin{array}{c}-\overline{-} \\
0.699 \\
231 \\
\end{array}$ & $\begin{array}{c}- \\
0.812 \\
321 \\
\end{array}$ & $\begin{array}{c}- \\
0.7079 \\
58\end{array}$ & $\begin{array}{c}- \\
0.4745 \\
24\end{array}$ & $\begin{array}{c}- \\
1.057 \\
397\end{array}$ & $\begin{array}{l}1.669 \\
294\end{array}$ & $\begin{array}{c}- \\
1.272 \\
899 \\
\end{array}$ \\
\hline $\begin{array}{l}\text { Kurtos } \\
\text { is }\end{array}$ & $\begin{array}{c}24.17 \\
154 \\
\end{array}$ & $\begin{array}{l}7.603 \\
920 \\
\end{array}$ & $\begin{array}{l}7.211 \\
122 \\
\end{array}$ & $\begin{array}{c}3.570 \\
364\end{array}$ & $\begin{array}{l}5.462 \\
489 \\
\end{array}$ & $\begin{array}{l}4.993 \\
187 \\
\end{array}$ & $\begin{array}{c}4.1286 \\
54 \\
\end{array}$ & $\begin{array}{l}3.985 \\
350 \\
\end{array}$ & $\begin{array}{l}5.611 \\
811 \\
\end{array}$ & $\begin{array}{c}21.84 \\
667 \\
\end{array}$ & $\begin{array}{c}10.17 \\
312 \\
\end{array}$ \\
\hline $\begin{array}{l}\text { Jarque } \\
\text {-Bera } \\
\end{array}$ & $\begin{array}{c}3090 . \\
296\end{array}$ & $\begin{array}{l}142.5 \\
859 \\
\end{array}$ & $\begin{array}{l}142.9 \\
312 \\
\end{array}$ & $\begin{array}{l}10.53 \\
165 \\
\end{array}$ & $\begin{array}{l}53.12 \\
951 \\
\end{array}$ & $\begin{array}{l}43.80 \\
622 \\
\end{array}$ & $\begin{array}{c}21.721 \\
24 \\
\end{array}$ & $\begin{array}{l}12.39 \\
939 \\
\end{array}$ & $\begin{array}{l}74.82 \\
216 \\
\end{array}$ & $\begin{array}{c}2427 . \\
024\end{array}$ & $\begin{array}{c}383.8 \\
178 \\
\end{array}$ \\
\hline $\begin{array}{l}\text { Probab } \\
\text { ility }\end{array}$ & $\begin{array}{l}0.000 \\
000\end{array}$ & $\begin{array}{l}0.000 \\
000\end{array}$ & $\begin{array}{c}0.000 \\
000\end{array}$ & $\begin{array}{l}0.005 \\
165 \\
\end{array}$ & $\begin{array}{c}0.000 \\
000\end{array}$ & $\begin{array}{c}0.000 \\
000\end{array}$ & $\begin{array}{c}0.0000 \\
19\end{array}$ & $\begin{array}{l}0.002 \\
030 \\
\end{array}$ & $\begin{array}{c}0.000 \\
000\end{array}$ & $\begin{array}{c}0.000 \\
000\end{array}$ & $\begin{array}{c}0.000 \\
000\end{array}$ \\
\hline Sum & $\begin{array}{c}0.471 \\
000\end{array}$ & $\begin{array}{c}- \\
0.070 \\
963 \\
\end{array}$ & $\begin{array}{l}1.353 \\
929\end{array}$ & $\begin{array}{c}0.481 \\
382\end{array}$ & $\begin{array}{l}1.389 \\
014\end{array}$ & $\begin{array}{c}0.808 \\
017\end{array}$ & $\begin{array}{c}0.5878 \\
42\end{array}$ & $\begin{array}{l}0.329 \\
709\end{array}$ & $\begin{array}{l}0.362 \\
012\end{array}$ & $\begin{array}{l}0.262 \\
876\end{array}$ & $\begin{array}{c}0.687 \\
172\end{array}$ \\
\hline $\begin{array}{l}\text { Sum } \\
\text { Sq. } \\
\text { Dev. } \\
\end{array}$ & $\begin{array}{l}1.210 \\
063\end{array}$ & $\begin{array}{l}0.039 \\
267\end{array}$ & $\begin{array}{l}1.230 \\
823\end{array}$ & $\begin{array}{l}0.363 \\
574\end{array}$ & $\begin{array}{l}0.488 \\
423\end{array}$ & $\begin{array}{l}0.232 \\
371\end{array}$ & $\begin{array}{c}0.2258 \\
75\end{array}$ & $\begin{array}{c}0.499 \\
130\end{array}$ & $\begin{array}{l}0.542 \\
373\end{array}$ & $\begin{array}{c}0.403 \\
430\end{array}$ & $\begin{array}{l}1.170 \\
923\end{array}$ \\
\hline Obs. & 159 & 159 & 159 & 159 & 159 & 159 & 159 & 159 & 159 & 159 & 159 \\
\hline \multicolumn{12}{|c|}{ Pre-crisis period: March 2003 - December 2006} \\
\hline Mean & $\begin{array}{l}0.026 \\
740\end{array}$ & $\begin{array}{c}- \\
0.004 \\
652 \\
\end{array}$ & $\begin{array}{l}0.034 \\
856\end{array}$ & $\begin{array}{l}0.016 \\
661\end{array}$ & $\begin{array}{c}0.020 \\
145\end{array}$ & $\begin{array}{c}0.009 \\
874\end{array}$ & $\begin{array}{c}0.0120 \\
73\end{array}$ & $\begin{array}{c}0.019 \\
545\end{array}$ & $\begin{array}{l}0.019 \\
406\end{array}$ & $\begin{array}{c}0.016 \\
031\end{array}$ & $\begin{array}{c}0.037 \\
237\end{array}$ \\
\hline $\begin{array}{l}\text { Std. } \\
\text { Dev. }\end{array}$ & $\begin{array}{c}0.0537 \\
25 \\
\end{array}$ & $\begin{array}{c}0.016 \\
808 \\
\end{array}$ & $\begin{array}{c}0.080 \\
896 \\
\end{array}$ & $\begin{array}{c}0.031 \\
709 \\
\end{array}$ & $\begin{array}{c}0.046 \\
241 \\
\end{array}$ & $\begin{array}{c}0.024 \\
189 \\
\end{array}$ & $\begin{array}{c}0.0235 \\
76 \\
\end{array}$ & $\begin{array}{c}0.033 \\
007 \\
\end{array}$ & $\begin{array}{l}0.032 \\
882 \\
\end{array}$ & $\begin{array}{c}0.033 \\
355 \\
\end{array}$ & $\begin{array}{c}0.065 \\
413 \\
\end{array}$ \\
\hline \multicolumn{12}{|c|}{ Crisis period: January 2007 - December 2012} \\
\hline Mean & $\begin{array}{c}- \\
0.0094 \\
99\end{array}$ & $\begin{array}{c}0.000 \\
788\end{array}$ & $\begin{array}{c}- \\
0.007 \\
475\end{array}$ & $\begin{array}{c}- \\
0.006 \\
084\end{array}$ & $\begin{array}{c}0.001 \\
632\end{array}$ & $\begin{array}{c}0.000 \\
528\end{array}$ & $\begin{array}{c}- \\
0.0007 \\
11\end{array}$ & $\begin{array}{c}- \\
0.0081 \\
36\end{array}$ & $\begin{array}{c}- \\
0.014 \\
042 \\
\end{array}$ & $\begin{array}{c}- \\
0.006 \\
073\end{array}$ & $\begin{array}{c}- \\
0.019 \\
087\end{array}$ \\
\hline $\begin{array}{l}\text { Std. } \\
\text { Dev. }\end{array}$ & $\begin{array}{c}0.1218 \\
83\end{array}$ & $\begin{array}{c}0.018 \\
663 \\
\end{array}$ & $\begin{array}{c}0.108 \\
912 \\
\end{array}$ & $\begin{array}{c}0.057 \\
722 \\
\end{array}$ & $\begin{array}{c}0.065 \\
997 \\
\end{array}$ & $\begin{array}{c}0.048 \\
059 \\
\end{array}$ & $\begin{array}{c}0.0469 \\
87 \\
\end{array}$ & $\begin{array}{c}0.0688 \\
73 \\
\end{array}$ & $\begin{array}{c}0.073 \\
404\end{array}$ & $\begin{array}{c}0.069 \\
672 \\
\end{array}$ & $\begin{array}{c}0.107 \\
261 \\
\end{array}$ \\
\hline \multicolumn{12}{|c|}{ Post-crisis Period: January 2013 - June 2016} \\
\hline Mean & $\begin{array}{c}- \\
0.0031 \\
61\end{array}$ & $\begin{array}{c}0.002 \\
066\end{array}$ & $\begin{array}{c}0.004 \\
140\end{array}$ & $\begin{array}{c}0.003 \\
094\end{array}$ & $\begin{array}{c}0.006 \\
993\end{array}$ & $\begin{array}{l}0.006 \\
279\end{array}$ & $\begin{array}{c}0.0008 \\
68\end{array}$ & $\begin{array}{c}- \\
0.0005 \\
87\end{array}$ & $\begin{array}{l}0.011 \\
327\end{array}$ & $\begin{array}{c}\overline{-} \\
0.001 \\
351\end{array}$ & $\begin{array}{c}0.003 \\
197\end{array}$ \\
\hline $\begin{array}{l}\text { Std. } \\
\text { Dev. }\end{array}$ & $\begin{array}{c}0.0001 \\
20 \\
\end{array}$ & $\begin{array}{c}0.005 \\
646\end{array}$ & $\begin{array}{c}0.036 \\
673 \\
\end{array}$ & $\begin{array}{c}0.042 \\
217 \\
\end{array}$ & $\begin{array}{c}0.044 \\
124\end{array}$ & $\begin{array}{l}0.031 \\
515\end{array}$ & $\begin{array}{c}0.0310 \\
38\end{array}$ & $\begin{array}{c}0.049 \\
136\end{array}$ & $\begin{array}{c}0.044 \\
697 \\
\end{array}$ & $\begin{array}{c}0.051 \\
284 \\
\end{array}$ & $\begin{array}{c}0.039 \\
469 \\
\end{array}$ \\
\hline
\end{tabular}

The coefficients of lagged variances and shock-square terms are all significant at $1 \%$, which means that the volatilities of these markets are time-varying (Table 2). The estimated parameters $\theta 1$ and $\theta 2$ of DCC processes are all significant at $1 \%$. The conditions that $\theta 1+\theta 2$ $<1$ are all satisfied. The results for the significance of the conditional variances prove that the market volatility changes during the explored period and confirms the conditional heteroscedasticity in the returns of the indices. The $\beta$ coefficients present long-term persistence while $\alpha$ coefficients measure the short-term persistence and the reaction of conditional volatility to market shocks. In Table 2 we prove that the $\alpha$ coefficient is law during the pre-crisis period. It increases its value for all of the explored indices during the crisis period and decreases during the post-crisis period. During the pre-crisis period the values of the $\alpha$ coefficients are higher than the values in the post-crisis period. This confirms the increased caution of the financial markets. The highest values of $\alpha$ coefficients are 
registered in the crisis period. We may conclude that in crisis the volatility of the indices is more sensitive to market shocks and dynamic than during the post-crisis period.

The autoregressive coefficient of volatility $\beta$ is higher during the crisis period than during the stable periods, either. The $\beta$ coefficients measure the persistence of conditional volatility to different market events and in the cases when the value of $\beta$ is high, volatility takes longer to vanish. The significance of $\theta 1$ and $\theta 2$ proves that the impact of lagged shocks and the impact of lagged dynamic correlations on dynamic conditional correlations are highly significant. These results support the dynamic conditional correlations model and allow us to reject the hypothesis of a constant correlation between the returns series.

Table 2. Results from the applied Bivariate DCC-GARCH model

\begin{tabular}{|c|c|c|c|c|c|c|c|c|c|c|}
\hline \multicolumn{11}{|c|}{ Pre-crisis period: March 2003 - December 2006} \\
\hline Par. & $\begin{array}{l}\text { SOFIX- } \\
\text { ATHE } \\
X\end{array}$ & $\begin{array}{l}\text { SOFIX- } \\
\text { BEL20 }\end{array}$ & $\begin{array}{l}\text { SOFIX- } \\
\text { BET }\end{array}$ & $\begin{array}{l}\text { SOFIX- } \\
\text { CAC40 }\end{array}$ & $\begin{array}{l}\text { SOFIX- } \\
\text { DAX }\end{array}$ & $\begin{array}{l}\text { SOFIX- } \\
\text { DJIA }\end{array}$ & $\begin{array}{l}\text { SOFIX- } \\
\text { FTSE10 } \\
0\end{array}$ & $\begin{array}{l}\text { SOFIX- } \\
\text { IBEX3 } \\
5\end{array}$ & $\begin{array}{l}\text { SOFIX- } \\
\text { ISEQ }\end{array}$ & $\begin{array}{l}\text { SOFIX- } \\
\text { PSI20 }\end{array}$ \\
\hline$\alpha(1)$ & $0.088^{* * *}$ & $\begin{array}{l}0.088^{*} \\
*\end{array}$ & $\begin{array}{l}0.088^{*} \\
*\end{array}$ & $\begin{array}{l}0.088^{*} \\
*\end{array}$ & $\begin{array}{l}0.088^{*} \\
*\end{array}$ & $\begin{array}{l}0.088^{*} \\
*\end{array}$ & $0.088^{* *}$ & $0.088^{* * *}$ & $0.088^{* *}$ & $\begin{array}{l}0.088^{*} \\
*\end{array}$ \\
\hline$\alpha(2)$ & $0.097^{* * *}$ & $\begin{array}{l}0.106 * \\
*\end{array}$ & $\begin{array}{l}0.102 * \\
*\end{array}$ & $\begin{array}{l}0.094 * \\
*\end{array}$ & $\begin{array}{l}0.112 * \\
*\end{array}$ & $0.124 *$ & $0.096^{* *}$ & $0.121^{*}$ & $\begin{array}{l}0.099 * * \\
*\end{array}$ & $\begin{array}{l}0.095 * \\
*\end{array}$ \\
\hline$\beta(1)$ & $0.931^{*}$ & $0.931 *$ & $0.931^{*}$ & $0.931^{*}$ & $0.931^{*}$ & $0.931^{*}$ & $0.931 *$ & $0.931^{*}$ & $0.931 *$ & $0.931 *$ \\
\hline$\beta(2)$ & 0.832 & 0.994* & $0.896^{*}$ & $0.902 *$ & $0.835^{*}$ & $\begin{array}{l}0.918^{*} \\
*\end{array}$ & $0.915 *$ & $0.891 *$ & $0.901 *$ & $0.825^{*}$ \\
\hline $\begin{array}{l}\Theta(1 \\
)^{2}\end{array}$ & $0.011^{*}$ & $0.023 *$ & $0.015^{*}$ & $0.035^{*}$ & $0.011^{*}$ & $0.028^{*}$ & $0.020^{*}$ & $0.025^{*}$ & $0.018^{*}$ & $0.037 *$ \\
\hline $\begin{array}{l}\Theta(2 \\
)\end{array}$ & $0.824 *$ & $0.925^{*}$ & $0.857^{*}$ & $0.927 *$ & $0.967 *$ & $0.805^{*}$ & $0.971 *$ & $0.834 *$ & $0.915^{*}$ & $0.795^{*}$ \\
\hline \multicolumn{11}{|c|}{$\begin{array}{l}\text { Crisis period: January } 2007 \text { - December } 2012 \\
\end{array}$} \\
\hline$\alpha(1)$ & $0.102^{*}$ & $0.102 *$ & $0.102 *$ & $0.102 *$ & $0.102^{*}$ & $0.102^{*}$ & $0.102 *$ & $0.102 *$ & $0.102 *$ & $0.102 *$ \\
\hline$\alpha(2)$ & $0.118^{*}$ & $0.154^{*}$ & $0.124 *$ & 0.094 & 0.174* & $\begin{array}{l}0.152 * \\
*\end{array}$ & $0.096^{*}$ & $0.116^{*}$ & $0.109^{* *}$ & $0.118^{*}$ \\
\hline$\beta(1)$ & $0.995^{*}$ & $0.995^{*}$ & $0.995^{*}$ & $0.995 *$ & $0.995^{*}$ & $0.995^{*}$ & $0.995 *$ & $0.995 *$ & $0.995^{*}$ & $0.995^{*}$ \\
\hline$\beta(2)$ & $0.874^{*}$ & $0.825 *$ & $0.915^{*}$ & $0.942 *$ & $0.926^{*}$ & $\begin{array}{l}0.915^{*} \\
*\end{array}$ & $0.894 * *$ & $0.948^{* *}$ & $0.879 * *$ & $0.918^{*}$ \\
\hline$\Theta(1$ & $0.018^{*}$ & $0.034 *$ & $0.028 *$ & $0.039 *$ & $0.152 *$ & $0.028^{*}$ & $0.034 *$ & $0.011 *$ & $0.013 *$ & $0.039^{*}$ \\
\hline$\Theta(2$ & $0.902^{*}$ & $0.892 *$ & $0.912^{*}$ & $0.915^{*}$ & $0.834 *$ & $0.905^{*}$ & $0.911^{*}$ & $0.907 *$ & $0.832 *$ & $0.902 *$ \\
\hline \multicolumn{11}{|c|}{ Post-crisis Period: January 2013 - June 2016} \\
\hline$\alpha(1)$ & $0.086^{*}$ & $0.086 *$ & $0.086^{*}$ & $0.086^{*}$ & $0.086^{*}$ & $0.086^{*}$ & $0.086^{*}$ & $0.086^{*}$ & $0.086^{*}$ & $0.086 *$ \\
\hline$\alpha(2)$ & $0.079^{*}$ & $0.082 *$ & $\begin{array}{l}0.091 * \\
*\end{array}$ & $\begin{array}{l}0.101 * \\
*\end{array}$ & $\begin{array}{l}0.086^{*} \\
*\end{array}$ & $\begin{array}{l}0.093 * \\
*\end{array}$ & 0.105 & 0.084 & $0.094 *$ & $0.090^{*}$ \\
\hline$\beta(1)$ & $0.898^{*}$ & $0.898^{*}$ & 0.898* & $0.898^{*}$ & $0.898^{*}$ & $0.898^{*}$ & $0.898^{*}$ & $0.898^{*}$ & $0.898^{*}$ & $0.898^{*}$ \\
\hline$\beta(2)$ & $0.912 *$ & $0.864 *$ & $0.906^{*}$ & 0.894 & 0.975 & $0.932 *$ & $0.906^{* *}$ & $0.861^{*}$ & 0.946 & $\begin{array}{l}0.857 * \\
*\end{array}$ \\
\hline$\Theta(1$ & $0.015^{*}$ & $0.021 *$ & $0.018^{*}$ & $0.032 *$ & $0.019^{*}$ & $0.012 *$ & $0.025^{*}$ & $0.019^{*}$ & $0.038^{*}$ & $0.028^{*}$ \\
\hline $\begin{array}{l}\Theta(2 \\
)\end{array}$ & $0.805^{*}$ & $0.912 *$ & $0.947 *$ & $0.835^{*}$ & $0.875^{*}$ & $0.912 *$ & $0.908^{*}$ & $0.846^{*}$ & $0.835^{*}$ & $0.812^{*}$ \\
\hline
\end{tabular}

Notes: $* * *, * *$ denote statistical significance at the $1 \%$ and $5 \%$ respectively

Figures $1-10$ present the dynamic conditional correlations of the Bulgarian stock index and the other examined stock indices. We should take into account the following observations: the dynamic correlation between SOFIX-ATHEX reaches its peak in 2009 when it is the peak of the Greek financial crisis and maintain high values during the sovereign debt crisis. This suggests that there is a strong transmission of negative information shocks during the sovereign debt crisis. The most significant is the dynamic correlation between SOFIX- DJIA with the highest peak between 2008-2009. The results show increased (stronger) correlation dynamics between the Bulgarian stock market and developed European countries and the United States during the crisis period, with overtaking information impact between the SOFIX-DAX. 


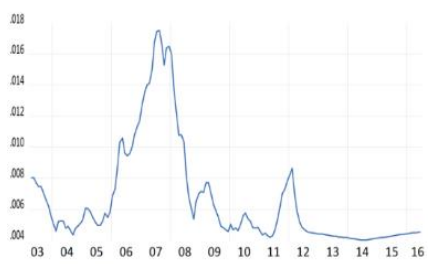

Fig. 1. Dynamic conditional correlations

\section{SOFIX-CAC40}

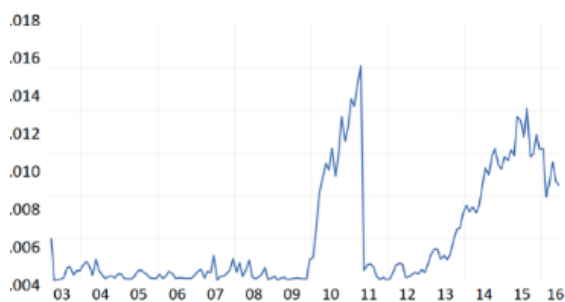

Fig. 2. Dynamic conditional correlations SOFIX-ATHEX

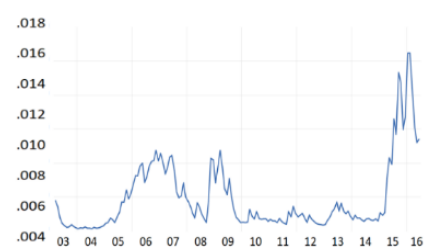

Fig. 3. Dynamic conditional correlations SOFIX-ISEQ

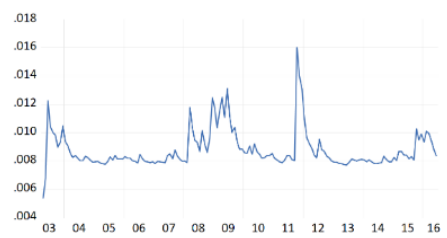

Fig. 4. Dynamic conditional correlations SOFIX-IBEX35

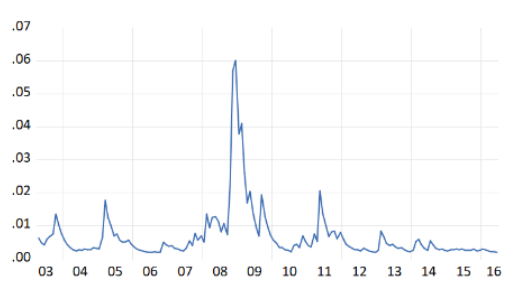

Fig. 5. Dynamic conditional correlations SOFIX-PSI20

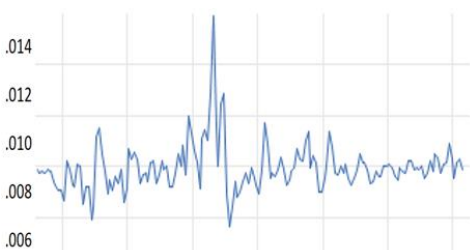

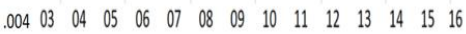

Fig. 6. Dynamic conditional correlations SOFIX- BEL20

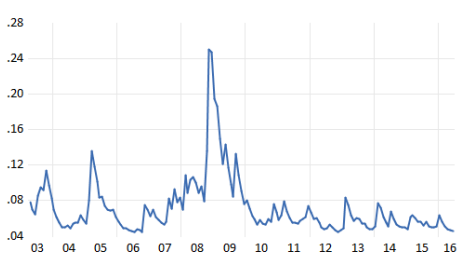

Fig. 7. Dynamic conditional correlations SOFIX- DJIA

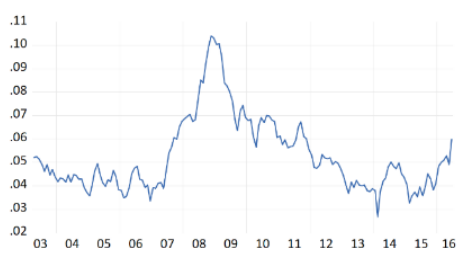

Fig. 8. Dynamic conditional correlations SOFIX- DAX

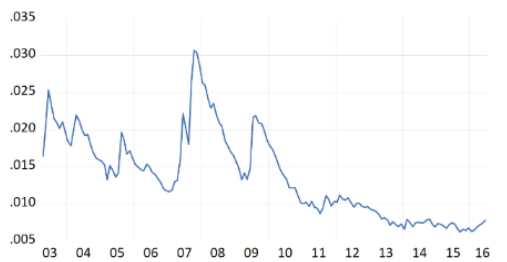

Fig. 9. Dynamic conditional correlations SOFIX-BET

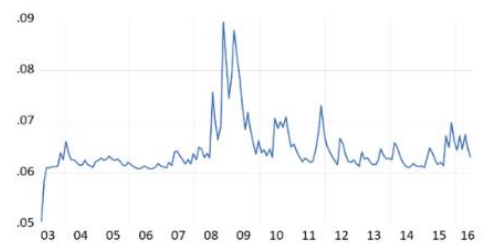

Fig. 10. Dynamic conditional correlations SOFIX-FTSE10

Table 3 presents the results of the contagion test. We prove that the dynamic condition correlations between examined indices increase from the periods with low volatility (precrisis period) to the period with high volatility (crisis period). We register the strongest negative information flow for Bulgarian and the US capital markets. The German DAX index also has a significant impact on the SOFIX return. Our results show that the financial 
contagion exists between Bulgarian and Romanian capital markets. The PIIGS block, which was most affected by the global financial crisis, has transferred negative shocks to the SOFIX.

Table 3. The Results from the Contagion Test

\begin{tabular}{|c|c|c|c|c|c|}
\hline \multicolumn{6}{|c|}{ Unadjusted Conditional Correlation } \\
\hline Relation & $\begin{array}{r}\text { Pre-crisis } \\
\rho_{i j}^{t}\end{array}$ & $\begin{array}{c}\text { Crisis period } \\
\rho_{i j}^{c}\end{array}$ & t-student & $\begin{array}{ll}\text { Change } & \text { of } \\
\text { correlation } & \\
\text { coefficients } & \end{array}$ & Contagion \\
\hline SOFIX-ATHEX & 0.492 & 0.715 & $11.14^{*}$ & $45.33 \%$ & YES \\
\hline SOFIX-BEL20 & 0.435 & 0.402 & 0.266 & $-7.59 \%$ & NO \\
\hline SOFIX-BET & 0.401 & 0.618 & $6.282 *$ & $54.11 \%$ & YES \\
\hline SOFIX-CAC40 & 0.358 & 0.418 & 0.083 & $16.76 \%$ & NO \\
\hline SOFIX-DAX & 0.374 & 0.608 & 9.084* & 62.56 & YES \\
\hline SOFIX-DJIA & 0.318 & 0.524 & $5.159 *$ & $64.77 \%$ & YES \\
\hline SOFIX-FTSE100 & 0.349 & 0.504 & $6.188^{*}$ & $44.41 \%$ & YES \\
\hline SOFIX-IBEX35 & 0.385 & 0.648 & $4.190^{*}$ & $44.41 \%$ & YES \\
\hline SOFIX-ISEQ & 0.486 & 0.682 & $8.154^{*}$ & $40.33 \%$ & YES \\
\hline SOFIX-PSI20 & 0.418 & 0.591 & $9.182 *$ & $41.39 \%$ & YES \\
\hline \multicolumn{6}{|c|}{ Adjusted Conditional Correlation } \\
\hline SOFIX-ATHEX & 0.232 & 0.352 & $7.315^{*}$ & $51.72 \%$ & YES \\
\hline SOFIX-BEL20 & 0.218 & 0.204 & 0.158 & $-6.42 \%$ & NO \\
\hline SOFIX-BET & 0.195 & 0.350 & $3.085^{*}$ & $79.48 \%$ & YES \\
\hline SOFIX-CAC40 & 0.182 & 0.218 & 0.018 & $19.78 \%$ & NO \\
\hline SOFIX-DAX & 0.186 & 0.358 & $6.084 *$ & $92.47 \%$ & YES \\
\hline SOFIX-DJIA & 0.192 & 0.376 & $3.794 *$ & $95.83 \%$ & YES \\
\hline SOFIX-FTSE100 & 0.159 & 0.284 & $3.042 *$ & $78.61 \%$ & YES \\
\hline SOFIX-IBEX35 & 0.197 & 0.326 & $2.381 *$ & $65.48 \%$ & YES \\
\hline SOFIX-ISEQ & 0.231 & 0.354 & $3.908^{*}$ & $53.24 \%$ & YES \\
\hline SOFIX-PSI 20 & 0.198 & 0.259 & $4.082 *$ & $30.80 \%$ & YES \\
\hline
\end{tabular}

Notes: $* * *, * *$ denote statistical significance at the $1 \%$ and $5 \%$ respectively

Table 4 shows the statistical significance of AR (1) for the whole period.

Table 4. The values of AR (1) in the TGARCH model for the whole period under examination

\begin{tabular}{|l|l|}
\hline Index & AR (1) for the whole examined period \\
\hline BEL 20 & -0.007471 \\
\hline CAC & 0.107091 \\
\hline DAX & 0.125258 \\
\hline IBEX 35 & 0.012454 \\
\hline DJIA & 0.281503 \\
\hline ATHEX & $0.948586^{*}$ \\
\hline ISEQ & $0.154979^{* * *}$ \\
\hline FTSE 100 & $-0.110156^{* * *}$ \\
\hline PSI 20 & $0.078225^{* *}$ \\
\hline SOFIX & $0.300833^{*}$ \\
\hline BET & $0.086118^{*}$ \\
\hline
\end{tabular}

Notes: $*, * *, * * *$ denote statistical significance at the $1 \%, 5 \%$ and $10 \%$ respectively

We register statistically significant AR (1) for the following countries: The United Kingdom, Greece, Ireland, Portugal, Romania, and Bulgaria. What is more, we can define these capital markets as inefficient and we can include them in three separate groups: developed, Eurozone's problem countries, and developing. The highest value of the AR (1) is registered for the Greek index ATHEX and this financial market can be determined as the most inefficient. In comparison, the US capital market can be defined as efficient due to the non-statistically significant values of AR (1). Based on the positive values of AR (1) of the Greek, Irish, Portuguese, Romanian, and Bulgarian indices, we can conclude that AR (1) gives a greater weight of the return of the previous period and therefore strengthens the established market trend. Additionally, the positive values of AR (1) lead to the accumulation and acceleration of the positive market trend. These results indicate an irrational acceptance and subsequent acceleration of the positive market trend because of the incoherent behavior of investors following the prospects for the development of those markets. The negative value of AR (1) for FTSI 100 index shows an opposite reaction to the subsequent positive market trends from the previous period. Based on this assumption we can conclude that the information influence from period $t$ is so incorporated in the market trend, that when it comes 
to lag $t-1$ the information losses all of its influence and becomes obsolete. We can separate the examined indices into two groups according to the values of the coefficient of persistence: Indices with relatively high market efficiency (the value of their coefficient of persistence is below 0,94); Indices with relatively low market efficiency (the value of their coefficient of persistence is higher than 0,94 ).

Table 5. The indices with relatively high market efficiency and their coefficients of persistence below 0,94 and leverage coefficients

\begin{tabular}{|c|c|c|}
\hline Index & Coefficient of persistence $<\mathbf{0 , 9 4}$ & Leverage coefficient \\
\hline DAX & 0.666247 & $0.293095^{* *}$ \\
\hline FTSE 100 & 0.756942 & $0.335537^{*}$ \\
\hline IBEX & 0.795043 & $0.268119^{*}$ \\
\hline CAC & 0.828806 & $0.769672^{* *}$ \\
\hline SOFIX & 0.849258 & -0.017604 \\
\hline PSI 20 & 0.863198 & -0.108970 \\
\hline BET & 0.895839 & $0.169299^{* *}$ \\
\hline DJIA & 0.901308 & $0.381560^{*}$ \\
\hline
\end{tabular}

Notes: *, ** denote statistical significance at the $1 \%$ and $5 \%$ respectively

Table 5 presents the values of the coefficient of persistence and leverage coefficient for the capital markets with relatively high market efficiency. The most efficient financial market in the group is the German one with the lowest value of the coefficient of persistence for the DAX index. Based on the above results, we can clearly distinguish developed countries like Germany and the UK and relatively new European stock markets - Bulgaria and Romania. The values of the leverage coefficient present how market volatility reacts depending on whether market impulses lead to positive or negative returns. Besides, the lowest positive value of the leverage coefficient is resisted for the Romanian BET index. This can be attributed to the fact that the market dynamics of Romanian stock exchange follow shortterm trends rather than stable, longer-term market trends.

Table 6. The indices with relatively low market efficiency and their coefficients of persistence higher than 0,94 and leverage coefficients

\begin{tabular}{|c|c|c|}
\hline Index & Coefficient of persistence $>\mathbf{0 , 9 4}$ & Leverage coefficient \\
\hline ATHEX & 1.838203 & $-0.692084 * * *$ \\
\hline BEL 20 & 1.015379 & $-0.064635^{*}$ \\
\hline ISEQ & 0.942459 & $0.078306^{*}$ \\
\hline Notes: $*, * * * *$ denote statistical significance at the 1\%, 5\% and 10\% respectively
\end{tabular}

Table 6 presents the values of the coefficient of persistence and leverage coefficient for the capital markets with relatively low market efficiency. We can conclude that Greek, Belgian, and Irish capital markets are relatively informationally inefficient markets compared to the other examined markets.

\section{Conclusion}

Our results show that a contagion across the Bulgarian capital market and eight capital markets exist during the financial crisis of 2008. We register the strongest contagion effects from US and German capital markets to the Bulgarian capital market. The obtained results indicate that the Bulgarian capital market is relatively integrated with the stock markets of Germany and the United States. That is the explanation of why the Bulgarian capital market is exposed to financial contagion effects from the US capital market and the capital markets of EU member states during the crisis period. As well as, correlation trends between bull and bear phases, indicating their dynamic nature and conditions. Overall, our results suggest that financial contagion from the US stock market and the capital markets of the developed 
European countries to the Bulgarian capital market occurs just before the financial crisis, but we find that the contagion is stronger during the crisis. Negative shocks from the PIIGS block have a strong impact on financial contagion during the sovereign debt crisis as well. On the other hand, the capital markets of the following countries: The United Kingdom, Greece, Ireland, Portugal, Romania, and Bulgaria can be defined as inefficient. The most efficient financial market in the group is the German one with the lowest value of the coefficient of persistence for the DAX index (0.666247).

\section{References}

1. Kenourgios, D., \& Dimitriou, D. (2014). Contagion effects of the global financial crisis in US and European real economy sectors. Panoeconomicus, 61(3), 275-288.

2. Stefanova, J. (2019). Prospects And Challenges Facing Frontier Stock Markets In The Western Balkans: Quo Vadis?, Financial Studies, 2, 6-36.

3. Cristi, S. and Cosmin, T. (2018). Financial systems integration: a literature survey, International Journal of Business Quantitative Economics and Applied Management Research, 5(5), 29-38.

4. Simeonov, S. (2020). Analiz na aktivnostta na osnovnite Iztochnoaziatski fondovi borsi (v perioda 2007 - 2019), e-Journal VFU, 13, 1- 25

5. Maria, P. A., Anuta, L. C. E., \& Simona, M. (2013). Testing the Long RangeDependence for the Central Eastern European and the Balkans Stock Markets. The Annals Of The University Of Oradea, 1113.

6. Badhani, K. N. (2015). Contagion or Integration: Dynamic Conditional Correlation between Indian and the US Equity Markets over Last Three Decades.

7. Dajcman, S., \& Festic, M. (2012). Interdependence between the Slovenian and European stock markets-A DCC-GARCH Analysis. Economic research-Ekonomska istraživanja, 25(2), 379-395.

8. Abuselidze, G., Reznik, N., Slobodianyk, A., \& Prokhorova, V. (2020). Global Financial Derivatives Market Development and Trading on the Example of Ukraine. In SHS Web of Conferences (Vol. 74, p. 05001). EDP Sciences.

9. Pfeiferova, D., \& Kucharova, I. (2020). Risks of collective investment undertakings in the context of global capital markets. In SHS Web of Conferences (Vol. 74, p. 01025). EDP Sciences.

10. Ters, K., \& Urban, J. (2018). Intraday dynamics of credit risk contagion before and during the euro area sovereign debt crisis: Evidence from central Europe. International Review of Economics \& Finance, 54, 123-142.

11. Harkmann, K. (2014). Stock Market Contagion from Western Europe to Central and Eastern Europe During the Crisis Years 2008-2012, Eastern European Economics, 52(3), 55-65,

12. Alexakis, C. \& Pappas, V., (2018). Sectoral dynamics of financial contagion in Europe - The cases of the recent crises episodes, Economic Modelling, Elsevier, 73(C), 222-239.

13. Mohti, W., Dionisio, A., Vieira, I. \& Ferreira, P., (2019). Financial contagion analysis in frontier markets: Evidence from the US subprime and the Eurozone debt crises, Physica A: Statistical Mechanics and its Applications, Elsevier, vol. 525(C), 1388-1398.

14. Horváth, R., Lyócsa, Š., \& Baumöhl, E. (2018). Stock market contagion in Central and Eastern Europe: unexpected volatility and extreme co-exceedance. The European Journal of Finance, 24(5), 391-412.

15. Caporin, M., Pelizzon, L., Ravazzolo, F., \& Rigobon, R. (2018). Measuring sovereign contagion in Europe. Journal of Financial Stability, 34, 150-181. 
16. Apergis, N., Christou, C., \& Kynigakis, I. (2019). Contagion across US and European financial markets: Evidence from the CDS markets. Journal of International Money and Finance, 96, 1-12.

17. Maneejuk, P., \& Yamaka, W. (2019). Predicting Contagion from the US Financial Crisis to International Stock Markets Using Dynamic Copula with Google Trends. Mathematics, 7(11), 1032.

18. Forbes, K., and Rigobon, R. (2002). No contagion, only interdependence: Measuring stock market comovements. Journal of Finance, 57(5), 2223-2261.

19. Trabelsi, M. A., \& Hmida, S. (2018). A dynamic correlation analysis of financial contagion: Evidence from the Eurozone stock markets. Entrepreneurial Business and Economics Review, 6(3), 129-141.

20. Savova, K. (2005). 1.9 THE FINANCIAL STATEMENTS OF ENTITIES AS STRATEGIC AND TACTICAL GUIDELINES IN THE DEVELOPMENT OF ACCOUNTING THEORY AND PRACTICE. Удосконалення обліку, контролю $i$ аналітичного забезпечення управління підприємством в умовах глобалізації економіки:[колективна монографія]/колектив, 4(34), 86. 